\title{
Convergence analysis of a variational quasi-reversibility approach for an inverse hyperbolic heat conduction problem
}

\author{
Vo Anh Khoa and Manh-Khang Dao \\ Dedicated to Professor Michael Victor Klibanov on his 70th birth anniversary
}

\begin{abstract}
We study a time-reversed hyperbolic heat conduction problem based upon the Maxwell-Cattaneo model of non-Fourier heat law. This heat and mass diffusion problem is a hyperbolic type equation for thermodynamics systems with thermal memory or with finite time-delayed heat flux, where the Fourier or Fick law is proven to be unsuccessful with experimental data. In this work, we show that our recent variational quasireversibility method for the classical time-reversed heat conduction problem, which obeys the Fourier or Fick law, can be adapted to cope with this hyperbolic scenario. We establish a generic regularization scheme in the sense that we perturb both spatial operators involved in the PDE. Driven by a Carleman weight function, we exploit the natural energy method to prove the well-posedness of this regularized scheme. Moreover, we prove the Hölder rate of convergence in the mixed $L^{2}-H^{1}$ spaces.
\end{abstract}

Keywords. Backward heat conduction problem, hyperbolic equation, quasi-reversibility method, energy estimates, Carleman weight, Hölder convergence.

2010 Mathematics Subject Classification. 65L70, 65L09, 65L60.

\section{Introduction}

\subsection{Statement of the inverse problem}

In this work, we are interested in the extension of our new quasi-reversibility $(\mathrm{QR})$ method in [21] for terminal boundary value problems. In this regard, we want to recover the initial distribution of an evolutionary equation, given the terminal data. This model is well known to be one of the classical problems in the field of inverse and ill-posed problems; cf. e.g. [12] for some background of typical models in

This work was supported by US Army Research Laboratory and US Army Research Office grant W911NF-19-1-0044. The work of V.A.K was also partly supported by the Research FoundationFlanders (FWO) in Belgium under the project named "Approximations for forward and inverse reaction-diffusion problems related to cancer models". The work of M.-K.D was supported by the Swedish Research Council grant (2016-04086). 
this research line. As to the applications of this model, having a reliable stable approximation of this backward-in-time problem is significantly helpful in many physical, biological and ecological contexts. Those are concretely involved in, e.g., the works $[4,10,22,25]$. In particular, the first contribution of this model being in mind relies on the heating/cooling transfer problem based upon the fact that sometimes, we want to measure the initial temperature of a material and our equipment only works at a given later time. Recently, this scenario has been extended to the case of a two-slab composite system with an ideal transmission condition in [25]. The second application we would like to address here is recovering blurry digital images acquired by camera sensors. This practical concern was initiated in [4] and has been scrutinized in the framework of source localization for brain tumor in [10]. In mathematical oncology, reconstructing the initial images of the tumor can be used for analyzing behaviors of cancer cells and then potentially for predicting the progression of neoplasms of early-stage patients. This initial reconstruction is also part of the so-called data assimilation procedure that has been of interest so far in weather forecasting (cf. [1]).

It is worth mentioning that considerations of such parabolic models indicate the use of the Fourier or Fick law. However, in some contexts of thermodynamics this typical law is proven to be unsuccessful with experimental data. In fact, any initial disturbance in a medium is propagated instantly when taking into account the parabolic case; cf. e.g. [5]. We also refer to the monograph [11] and some impressive works [20,27], where some electromechanical models were studied to unveil this non-standard incompatibility. In order to avoid the phenomenon of infinite propagation, the Cattaneo-Vernotte law was derived, proposing that the parabolic case should be upgraded to a hyperbolic form. In terms of PDEs, it means one should consider

$$
u_{t t}+u_{t}-\Delta u=0 \quad \text { in } \Omega \times(0, T),
$$

where $T>0$ is the final time and $\Omega \subset \mathbb{R}^{d}(d=1,2,3)$ is a regular bounded domain of interest with a sufficiently smooth boundary. In electrodynamics, equation (1) is the same as the telegrapher's equation derived from the Maxwell equation. That is why one usually refers (1) to as the Maxwell-Cattaneo model.

In this work, we investigate a generalized model of (1) due to our mathematical interest. We assume to look for $u(x, t): \Omega \times(0, T) \rightarrow \mathbb{R}$ satisfying the following evolutionary equation:

$$
u_{t t}+u_{t}-\Delta u-\Delta u_{t}=0 \quad \text { in } \Omega \times(0, T) .
$$

In the studies of the motion of viscoelastic materials, this is well-known to be the linear strongly damped wave equation, where the weak and strong damping terms 
$\left(u_{t}\right.$ and $\left.-\Delta u_{t}\right)$ are altogether involved in the PDE. Cf. [3] and references cited therein, the solution $u$ in that setting can be viewed as a displacement, whilst it is a temperature field in the context of thermodynamics we have mentioned above. Going back to the heat context, we note that the underlying equation (2) is also related to the so-called Gurtin-Pipkin model, which reads as

$$
\theta_{t}=\int_{0}^{t} \kappa(t-s) \theta_{x x}(s) d s .
$$

When the kernel $\kappa$ is a constant, (3) becomes an integrated wave equation after differentiation in time. If $\kappa(t)=e^{-t}$, one has the weakly damped wave equation $u_{t t}+u_{t}-u_{x x}=0$. Furthermore, when $\kappa(t)=\delta(t)$, we get back to the classical heat equation. Therefore, we can conclude that our mathematical analysis for (2) really works for many distinctive physical applications at the same time.

To complete the time-reversed model, we endow (2) with the following boundary and terminal conditions:

$$
\left\{\begin{array}{l}
u(x, t)=0 \quad \text { on } \partial \Omega \times(0, T), \\
u(x, T)=f_{0}(x), u_{t}(x, T)=f_{1}(x) \text { in } \Omega .
\end{array}\right.
$$

Hence, (2) and (4) form our terminal boundary value problem. As to the illposedness of this problem, we refer to [26] for proof of its natural instability using the spectral approach.

\subsection{Historical remarks and contributions of the paper}

In the context of the time-reversed parabolic problem, many regularization schemes were extensively designed in order to circumvent its natural ill-posedness. Inverse problems for parabolic equations with memory effects were investigated in $[2,19]$. Since the aim of this work is extending our new QR method in [21] to the hyperbolic heat conduction scenario, we would like to address some existing literature just on the QR topic close to the explicit technique we are developing. Meanwhile, some implicit QR methods for the backward heat conduction problem can be referred to the works $[6,7,17,18]$. The "implicit" here means that the scheme is designed by perturbing the kernel of the unbounded operator itself. Another QR-based approaches using minimization were studied in e.g. [14, 15].

The very first idea about quasi-reversibility of time-reversed parabolic problems was established by Lattès and Lions in the monograph [16] when they used a fourth-order spatial perturbation to stabilize the Laplace operator involved in the classical time-reversed parabolic equation. Motivated by this approach, several modifications and variants were constructed and analyzed through five decades, 
which makes this method considerable in the field of inverse and ill-posed problems. For example, we mention here the pioneering work [23], where a third-order operator in space and time was proposed to obtain a regularization scheme in the form of a pseudoparabolic equation. Recently, Kaltenbacher et al. [13] has used a nonlocal perturbing operator in time with fractional order to regularize the illposed problem.

Our newly developed QR method follows the original idea of Lattès and Lions, i.e. we only use the spatial perturbation to stabilize the unbounded spatial operator. The key ingredient of our method lies in the fact that we use the perturbation operator to turn the inverse problem into a forward-like problem involving the stabilized operator. This notion has been studied in a spectral form in our recent work [24]. As a follow-up, we generalize this method in [21] by the establishment of conditional estimates for both the perturbation and stabilized operators. Driven by a Carleman weight function, we further apply the conventional energy method to show both well-posedness of the regularized system and error bounds. This way allows us to derive the scheme in the finite element setting and prove the error estimates in the finite-dimensional space. This will be our next target work in the future.

This work is the first time we extend our new method to the ill-posed problem (2) and (4). Intuitively, we construct in section 2 a generic regularized system in the sense that we perturb all the spatial terms $-\Delta u$ and $-\Delta u_{t}$. We then use the conditional estimates established in [21] to obtain the Hölder rate of convergence in section 4. Besides, well-posedness of the regularized system is considered in section 3 using a priori estimates and compactness arguments.

\section{A variational quasi-reversibility framework}

To this end, $\langle\cdot, \cdot\rangle$ indicates either the scalar product in $L^{2}(\Omega)$ or the dual pairing of a continuous linear functional and an element of a function space. Also, $\|\cdot\|$ is the norm in $L^{2}(\Omega)$. Different inner products and norms should be written as $\langle\cdot, \cdot\rangle_{X_{1}}$ and $\|\cdot\|_{X_{2}}$, respectively, where $X_{1}$ is a certain Hilbert space and $X_{2}$ is a Banach space. In the sequel, we denote $\varepsilon \in(0,1)$ by the noise level of the terminal data $f_{0}, f_{1}$ in (4). Any constant $C>0$ may vary from line to line. We usually indicate its dependencies if necessary.

We introduce an auxiliary function $\gamma:=\gamma(\varepsilon) \geq 1$ satisfying $\lim _{\varepsilon \rightarrow 0} \gamma(\varepsilon)=\infty$.

Definition 2.1 (perturbing operator). The linear mapping $\mathbf{Q}_{\varepsilon}: L^{2}(\Omega) \rightarrow L^{2}(\Omega)$ is said to be a family of $\varepsilon$-dependent perturbing operator if there exist a function 
space $\mathbb{W} \subset L^{2}(\Omega)$ and a noise-independent constant $C_{0}>0$ such that

$$
\left\|\mathbf{Q}_{\varepsilon} u\right\| \leq C_{0}\|u\|_{\mathbb{W}} / \gamma(\varepsilon) \quad \text { for any } u \in \mathbb{W} .
$$

Definition 2.2 (stabilized operator). The linear mapping $\mathbf{P}_{\varepsilon}: L^{2}(\Omega) \rightarrow L^{2}(\Omega)$ is said to be a family of $\varepsilon$-dependent stabilized operator if there exists a noiseindependent constant $C_{1}>0$ such that

$$
\left\|\mathbf{P}_{\varepsilon} u\right\| \leq C_{1} \log (\gamma(\varepsilon))\|u\| \quad \text { for any } u \in L^{2}(\Omega) .
$$

In this work, we start off with the generic approach of this modified version by stabilizing both two terms $-\Delta u$ and $-\Delta u_{t}$. By choosing the stabilization $\mathbf{P}_{\varepsilon}=$ $2 \Delta+\mathbf{Q}_{\varepsilon}$, our regularized equation is of the following form:

$$
u_{t t}^{\varepsilon}+u_{t}^{\varepsilon}+\Delta u^{\varepsilon}+\Delta u_{t}^{\varepsilon}=\mathbf{P}_{\varepsilon} u^{\varepsilon}+\mathbf{P}_{\varepsilon} u_{t}^{\varepsilon} \quad \text { in } \Omega \times(0, T) .
$$

It is worth noting that these perturbing and stabilized operators are constructed with respect to the variable $x$ only. Our main purpose in this finding is that we are able to obtain the convergence analysis of a family of regularization schemes based upon some particular conditional estimates of such perturbations and stabilizations. To qualify the convergence of such regularization schemes, the conditional estimates (5) and (6) are particularly needed. Some particular choices of these perturbing and stabilized operators shall be discussed in Remark 2.4.

Now we complete our regularized problem. Since in real-world applications the terminal data are usually noisy, we endow (7) with the following boundary and terminal conditions:

$$
\left\{\begin{array}{l}
u^{\varepsilon}(x, t)=0 \quad \text { on } \partial \Omega \times(0, T) \\
u^{\varepsilon}(x, T)=f_{0}^{\varepsilon}(x), u_{t}^{\varepsilon}(x, T)=f_{1}^{\varepsilon}(x) \text { in } \Omega .
\end{array}\right.
$$

In (8), we assume to have a noise level $\varepsilon \in(0,1)$ such that

$$
\left\|u^{\varepsilon}(\cdot, T)-u(\cdot, T)\right\|_{H^{1}(\Omega)}+\left\|u_{t}^{\varepsilon}(\cdot, T)-u_{t}(\cdot, T)\right\| \leq \varepsilon
$$

To validate our mathematical analysis below, we suppose that $f_{0}, f_{0}^{\varepsilon} \in H^{1}(\Omega)$ and $f_{1}, f_{1}^{\varepsilon} \in L^{2}(\Omega)$.

Remark 2.3. By the standard Fredholm theory, there exist

- a non decreasing sequence of nonegative real numbers $\left\{\mu_{k}\right\}_{k=1}^{\infty}$ that tends to $+\infty$ as $k \rightarrow \infty$, 
- a Hilbert basis $\left\{\phi_{k}\right\}_{k=1}^{\infty}$ of $L^{2}(\Omega)$ such that $\phi_{k} \in H_{0}^{1}(\Omega)$ such that

$$
\int_{\Omega} \nabla \phi_{k} \cdot \nabla \phi d x=\mu_{k} \int_{\Omega} \phi_{k} \phi d x \quad \text { for all } \phi \in H_{0}^{1}(\Omega) .
$$

Remark 2.4. By Remark 2.3, we can take

$$
\mathbf{Q}_{\varepsilon} h=2 \sum_{\mu_{p} \geq \frac{1}{2} \log (\gamma)} \mu_{p}\left\langle h, \phi_{p}\right\rangle \phi_{p} \quad \text { for } \gamma>1 .
$$

It is immediate to see that the conditional one (5) holds for $\mathbb{W}=\mathbb{G}_{1,1}(\Omega)$ and $C_{0}=2$ by using the Parseval identity. Here, cf. [24], we denote $\mathbb{G}_{\sigma, \alpha}(\Omega)$ by the Gevrey class of functions of order $\gamma>0$ and index $\alpha>0$ :

$$
\mathbb{G}_{\sigma, \alpha}(\Omega):=\left\{u \in L^{2}(\Omega): \sum_{p=0}^{\infty} \mu_{p}^{\alpha} e^{2 \sigma \mu_{p}}\left|\left\langle u, \phi_{p}\right\rangle\right|^{2}<\infty\right\} .
$$

By (10) we obtain

$$
\begin{aligned}
\mathbf{P}_{\varepsilon} h & =2 \Delta h+\mathbf{Q}_{\varepsilon} h=-2 \sum_{p \in \mathbb{N}} \mu_{p}\left\langle h, \phi_{p}\right\rangle \phi_{p}+2 \sum_{\mu_{p} \geq \frac{1}{2} \log (\gamma)} \mu_{p}\left\langle h, \phi_{p}\right\rangle \phi_{p} \\
& =-2 \sum_{\mu_{p}<\frac{1}{2} \log (\gamma)} \mu_{p}\left\langle h, \phi_{p}\right\rangle \phi_{p} .
\end{aligned}
$$

Thereupon, this $\mathbf{P}_{\varepsilon}$ satisfies the conditional estimate (6) with $C_{1}=1$.

\section{Well-posedness of the regularized system (7)-(8)}

Let $v^{\varepsilon}(x, t):=e^{\rho(t-T)} u^{\varepsilon}(x, t)$ where $\rho>1$ is a constant chosen later. Then (7)(8) become

$$
v_{t t}^{\varepsilon}+(1-2 \rho) v_{t}^{\varepsilon}+\left(\rho^{2}-\rho\right) v^{\varepsilon}+(1-\rho) \Delta v^{\varepsilon}+\Delta v_{t}^{\varepsilon}=(1-\rho) \mathbf{P}_{\varepsilon} v^{\varepsilon}+\mathbf{P}_{\varepsilon} v_{t}^{\varepsilon} \text { in } \Omega \times(0, T)
$$

and the boundary and terminal conditions:

$$
\left\{\begin{array}{l}
v^{\varepsilon}(x, t)=0 \quad \text { on } \partial \Omega \times(0, T), \\
v^{\varepsilon}(x, T)=f_{0}^{\varepsilon}(x), v_{t}^{\varepsilon}(x, T)=\rho f_{0}^{\varepsilon}(x)+f_{1}^{\varepsilon}(x) \quad \text { in } \Omega .
\end{array}\right.
$$

Remark 3.1. The most important difficult need to solve the regularized system (7)(8) lies in the term $+\Delta u^{\varepsilon}$, which is bad for our energy estimations for $u^{\varepsilon}$. More 
precisely, the sign of this term is technically impeding the energy of the gradient term and eventually, it ruins our mathematical analysis in this section. In order to circumvent this, we consider the system (12)-(13) for $v^{\varepsilon}$, which is equivalent to the regularized system (7)-(8). Since $\rho>1$, then $(1-\rho) \Delta v^{\varepsilon}$ becomes a "good term" and we shall use its effect to obtain the energy estimate for $v^{\varepsilon}$ in Theorem 3.5. This leads us to the well-posedness of (12)-(13) as well as that of (7)-(8).

Definition 3.2. A function $v \in L^{2}\left(0, T ; H_{0}^{1}(\Omega)\right)$ with $v_{t} \in L^{2}\left(0, T ; H^{1}(\Omega)\right)$ and $v_{t t} \in L^{2}\left(0, T ; H^{-1}(\Omega)\right)$ is a weak solution of (12)-(13) if for every test function $\varphi \in H_{0}^{1}(\Omega)$, it holds that

$$
\begin{aligned}
& \left\langle v_{t t}(t), \varphi\right\rangle_{H^{-1}, H_{0}^{1}}+(1-2 \rho)\left\langle v_{t}(t), \varphi\right\rangle+\left(\rho^{2}-\rho\right)\langle v(t), \varphi\rangle \\
& +(\rho-1)\langle\nabla v(t), \nabla \varphi\rangle-\left\langle\nabla v_{t}(t), \nabla \varphi\right\rangle=(1-\rho)\left\langle\mathbf{P}_{\varepsilon} v(t), \varphi\right\rangle+\left\langle\mathbf{P}_{\varepsilon} v_{t}(t), \varphi\right\rangle
\end{aligned}
$$

for a.e. $t \in(0, T)$, and $v(x, T)=f_{0}^{\varepsilon}(x), v_{t}(x, T)=\rho f_{0}^{\varepsilon}(x)+f_{1}^{\varepsilon}(x)$ in $\Omega$.

Our proof of well-posedness relies on the conventional Galerkin method. This means that we construct solution of some finite-dimensional approximations to (14).

Lemma 3.3. For any positive $n$, there exist $n$ absolutely continuous functions $y_{k}^{n}$ : $[0, T] \rightarrow \mathbb{R}, k=1, \ldots, n$ and a function $v_{n} \in L^{2}\left(0, T ; H_{0}^{1}(\Omega)\right)$, where $\partial_{t} v_{n} \in$ $L^{2}\left(0, T ; H^{1}(\Omega)\right)$ and $\partial_{t t} v_{n} \in L^{2}\left(0, T ; H^{-1}(\Omega)\right)$, of the form

$$
v_{n}(x, t)=\sum_{k=1}^{n} y_{k}^{n}(t) \phi_{k}(x),
$$

such that for $k=1, \ldots, n$

$$
\left\{\begin{array}{l}
y_{k}^{n}(T)=\int_{\Omega} f_{0}^{\varepsilon}(x) \phi_{k}(x) d x=: g_{0 k}(T), \\
\partial_{t} y_{k}^{n}(T)=\int_{\Omega}\left(\rho f_{0}^{\varepsilon}(x)+f_{1}^{\varepsilon}(x)\right) \phi_{k}(x) d x=: g_{1 k}(T),
\end{array}\right.
$$

and $v_{n}$ satisfies

$$
\begin{aligned}
& \int_{\Omega} \partial_{t t} v_{n}(t) \phi_{k} d x+(1-2 \rho) \int_{\Omega} \partial_{t} v_{n}(t) \phi_{k} d x+\left(\rho^{2}-\rho\right) \int_{\Omega} v_{n}(t) \phi_{k} d x \\
& +(\rho-1) \int_{\Omega} \nabla v_{n}(t) \cdot \nabla \phi_{k} d x-\int_{\Omega} \nabla \partial_{t} v_{n}(t) \cdot \nabla \phi_{k} d x \\
& =(1-\rho) \int_{\Omega} \mathbf{P}_{\varepsilon} v(t) \phi_{k} d x+\int_{\Omega} \mathbf{P}_{\varepsilon} \partial_{t} v(t) \phi_{k} d x .
\end{aligned}
$$


Proof. By the properties of $\left\{\phi_{i}\right\}_{i=1}^{\infty}$ in Remark 2.3, (17) is equivalent to

$$
\begin{aligned}
& \partial_{t t} y_{k}^{n}(t)+\left(1-2 \rho-\mu_{k}\right) \partial_{t} y_{k}^{n}(t)+\left(\rho^{2}+\left(\mu_{k}-1\right) \rho-\mu_{k}\right) y_{k}^{n}(t) \\
& =(1-\rho) \sum_{i=0}^{n} y_{i}^{n}(t)\left\langle\mathbf{P}_{\varepsilon} \phi_{i}, \phi_{k}\right\rangle+\sum_{i=0}^{n} \partial_{t} y_{i}^{n}(t)\left\langle\mathbf{P}_{\varepsilon} \phi_{i}, \phi_{k}\right\rangle \text { for a.e. } t \in(0, T) .
\end{aligned}
$$

Let $z_{k}^{n}=\frac{d}{d t} y_{k}^{n}$, it follows from (16) and (18) that

$$
\frac{d}{d t}\left[\begin{array}{l}
y_{k}^{n} \\
z_{k}^{n}
\end{array}\right]+A_{k}\left[\begin{array}{c}
y_{k}^{n} \\
z_{k}^{n}
\end{array}\right]=F_{k}, \quad\left[\begin{array}{c}
y_{k}^{n}(T) \\
z_{k}^{n}(T)
\end{array}\right]=\left[\begin{array}{c}
g_{0 k}(T) \\
g_{1 k}(T)
\end{array}\right],
$$

where $F_{k}^{n}=\left[0,(1-\rho) \sum_{i=0}^{n} y_{k}^{n}\left\langle\mathbf{P}_{\varepsilon} \phi_{i}, \phi_{k}\right\rangle+\sum_{i=0}^{n} z_{k}^{n}\left\langle\mathbf{P}_{\varepsilon} \phi_{i}, \phi_{k}\right\rangle\right]^{T}$ and

$$
A_{k}=\left[\begin{array}{cc}
0 & 1 \\
\rho^{2}+\left(\mu_{k}-1\right) \rho-\mu_{k} & 1-2 \rho-\mu_{k}
\end{array}\right] .
$$

Consider $w_{k}^{n}:=\left[y_{k}^{n}, z_{k}^{n}\right]^{T}$. We thus obtain the following integral equation:

$$
w_{k}^{n}(t)=w_{k}^{n}(T)+A_{k} \int_{t}^{T} w_{k}^{n}(s) d s-\int_{t}^{T} F_{k}^{n}(s) d s .
$$

Hereafter, we denote by $w_{n}:=\left[w_{1}^{n}, \ldots, w_{n}^{n}\right]:[0, T] \rightarrow \mathbb{R}^{2 n}$. The integral equation (19) can be rewritten as $w_{n}=H\left[w_{n}\right]$, where the same notation as $w_{n}$ is applied to $H$ with $H_{k}^{n}$ being the right-hand side of (19). To be more specific,

$$
H_{k}^{n}\left[w_{n}\right](t):=w_{k}^{n}(T)+A_{k} \int_{t}^{T} w_{k}^{n}(s) d s-\int_{t}^{T} F_{k}^{n}(s) d s .
$$

Define the norm in $Y=C\left([0, T] ; \mathbb{R}^{2 n}\right)$ as follows:

$$
\|c\|_{Y}:=\sup _{t \in[0, T]} \sum_{j=1}^{n}\left|c_{j}(t)\right| \quad \text { with } c=\left[c_{j}\right] \in C\left([0, T] ; \mathbb{R}^{2 n}\right) .
$$

We claim that there exists $n_{0} \in \mathbb{N}^{*}$ such that the operator

$$
H^{\left(n_{0}\right)}:=H\left[H^{\left(n_{0}-1\right)}\right]: Y \rightarrow Y
$$

is a contraction mapping. In other words, we find $K \in[0,1)$ such that

$$
\left\|H^{\left(n_{0}\right)}\left[w_{n}\right]-H^{\left(n_{0}\right)}\left[\tilde{w}_{n}\right]\right\|_{Y} \leq K\left\|w_{n}-\tilde{w}_{n}\right\|_{Y} \quad \text { for any } w_{n}, \tilde{w}_{n} \in Y .
$$


This can be done by induction. Indeed, let us observe that

$$
\begin{aligned}
& \left|H_{k}^{n}\left[w_{n}\right](t)-H_{k}^{n}\left[\tilde{w}_{n}\right](t)\right| \leq \int_{t}^{T}\left|A_{k}\right|\left|w_{k}^{n}(s)-\tilde{w}_{k}^{n}(s)\right| d s \\
& +\int_{t}^{T}\left(C_{1} C \log (\gamma) \sum_{i=1}^{n}\left(|1-\rho|\left|y_{i}^{n}(s)-\tilde{y}_{i}^{n}(s)\right|+\left|z_{i}^{n}(s)-\tilde{z}_{i}^{n}(s)\right|\right)\right) d s \\
& \leq \int_{t}^{T}\left(\left|A_{k}\right|+C_{1} C \log (\gamma)(\rho-1)\right)\left|w_{k}^{n}(s)-\tilde{w}_{k}^{n}(s)\right| d s \\
& \leq\left(\left|A_{k}\right|+C_{1} C \log (\gamma)(\rho-1)\right)(T-t)\left\|w_{n}-\tilde{w}_{n}\right\|_{Y},
\end{aligned}
$$

aided by the conditional estimate (6). Here, we indicate $C=\max _{i} C\left(\left\|\phi_{i}\right\|_{H_{0}^{1}(\Omega)}\right)>$ 0 . Furthermore, for any $m \in \mathbb{N}^{*}$

$$
\begin{aligned}
& \left|\left(H_{k}^{n}\right)^{(m)}\left[w_{n}\right](t)-\left(H_{k}^{n}\right)^{(m)}\left[\tilde{w}_{n}\right](t)\right| \\
& \leq \int_{t}^{T}\left(\left|A_{k}\right|+C_{1} C \log (\gamma)(\rho-1)\right)\left|\left(H_{k}^{n}\right)^{(m-1)}\left[w_{n}\right](s)-\left(H_{k}^{n}\right)^{(m-1)}\left[\tilde{w}_{n}\right](s)\right| d s,
\end{aligned}
$$

and it follows by induction that

$$
\begin{aligned}
& \left|\left(H_{k}^{n}\right)^{(m)}\left[w_{n}\right](t)-\left(H_{k}^{n}\right)^{(m)}\left[\tilde{w}_{n}\right](t)\right| \\
& \leq\left(\left|A_{k}\right|+C_{1} C \log (\gamma)(\rho-1)\right)^{m} \frac{(T-t)^{m}}{m !}\left\|w_{n}-\tilde{w}_{n}\right\|_{Y} .
\end{aligned}
$$

Therefore, we obtain

$$
\begin{aligned}
& \left\|H^{(m)}\left[w_{n}\right]-H^{(m)}\left[\tilde{w}_{n}\right]\right\|_{Y} \\
& \leq\left\|w_{n}-\tilde{w}_{n}\right\|_{Y} \frac{T^{m}}{m !} \sum_{k=1}^{n}\left(\left|A_{k}\right|+C_{1} C \log (\gamma)(\rho-1)\right)^{m} .
\end{aligned}
$$

Since the left-hand side tends to 0 as $m \rightarrow \infty$, we can find a sufficiently large $m_{0}$ such that

$$
\frac{T^{m_{0}}}{m_{0} !} \sum_{k=1}^{n}\left(\left|A_{k}\right|+C_{1} C \log (\gamma)(\rho-1)\right)^{m_{0}}<1 .
$$

The claim is proved and by the Banach fixed-point argument, there exists a unique solution $\bar{w}_{n} \in Y$ such that $H^{\left(m_{0}\right)}\left[\bar{w}_{n}\right]=\bar{w}_{n}$. Finally, since $H^{\left(m_{0}\right)}\left[H\left[\bar{w}_{n}\right]\right]=$ $H\left[H^{\left(m_{0}\right)}\left[\bar{w}_{n}\right]\right]=H\left[\bar{w}_{n}\right]$, then the integral equation (19) admits a unique solution in $Y$. Hence, we complete the proof of the lemma. 
Remark 3.4. By Lemma 3.3, it is easy to check that there exists a constant $C>0$ such that

$$
\left\|\partial_{t} v_{n}^{\varepsilon}(T)\right\|^{2},\left\|v_{n}^{\varepsilon}(T)\right\|^{2},\left\|\nabla v_{n}^{\varepsilon}(T)\right\|^{2} \leq C \quad \text { for all } n \in \mathbb{N} .
$$

Theorem 3.5. Assume (9) holds. For each $\varepsilon>0$, the regularized system (12)-(13) admits a unique weak solution $v^{\varepsilon}$ in the sense of Definition 3.2.

Proof. To prove the existence, we need to derive some energy estimates for approximate solution $v_{n}^{\varepsilon}$. Thanks to Lemma 3.3, we have $\partial_{t} v_{n}^{\varepsilon} \in C\left([0,1] ; H^{1}(\Omega)\right)$. Multiplying (17) by $\partial_{t} y_{k}^{n}(t)$, summing for $k=1, \ldots, n$ and using the formula (15) for $v_{n}^{\varepsilon}$, we get

$$
\begin{aligned}
& \int_{\Omega} \partial_{t t} v_{n}^{\varepsilon}(t) \partial_{t} v_{n}^{\varepsilon}(t) d x+(1-2 \rho) \int_{\Omega}\left|\partial_{t} v_{n}^{\varepsilon}(t)\right|^{2} d x+\left(\rho^{2}-\rho\right) \int_{\Omega} v_{n}^{\varepsilon}(t) \partial_{t} v_{n}^{\varepsilon}(t) d x \\
& +(\rho-1) \int_{\Omega} \nabla v_{n}^{\varepsilon}(t) \cdot \nabla \partial_{t} v_{n}^{\varepsilon}(t) d x-\int_{\Omega}\left|\nabla \partial_{t} v_{n}^{\varepsilon}(t)\right|^{2} d x \\
& =(1-\rho) \int_{\Omega} \mathbf{P}_{\varepsilon}\left(v_{n}^{\varepsilon}(t)\right) \partial_{t} v_{n}^{\varepsilon}(t) d x+\int_{\Omega} \mathbf{P}_{\varepsilon}\left(\partial_{t} v_{n}^{\varepsilon}(t)\right) \partial_{t} v_{n}^{\varepsilon}(t) d x
\end{aligned}
$$

This implies

$$
\begin{aligned}
& \frac{1}{2} \partial_{t}\left[\left\|\partial_{t} v_{n}^{\varepsilon}(t)\right\|^{2}+\left(\rho^{2}-\rho\right)\left\|v_{n}^{\varepsilon}(t)\right\|^{2}+(\rho-1)\left\|\nabla v_{n}^{\varepsilon}(t)\right\|^{2}\right] \\
& -(2 \rho-1) \int_{\Omega}\left|\partial_{t} v_{n}^{\varepsilon}(t)\right|^{2} d x-\int_{\Omega}\left|\nabla \partial_{t} v_{n}^{\varepsilon}(t)\right|^{2} d x \\
& =(1-\rho) \int_{\Omega} \mathbf{P}_{\varepsilon}\left(v_{n}^{\varepsilon}(t)\right) \partial_{t} v_{n}^{\varepsilon}(t) d x+\int_{\Omega} \mathbf{P}_{\varepsilon}\left(\partial_{t} v_{n}^{\varepsilon}(t)\right) \partial_{t} v_{n}^{\varepsilon}(t) d x \\
& \geq(1-\rho) C_{1} \log (\gamma)\left(\left\|v_{n}^{\varepsilon}(t)\right\|_{H^{1}(\Omega)}^{2}+\left\|\partial_{t} v_{n}^{\varepsilon}(t)\right\|^{2}\right)-C_{1} \log (\gamma)\left\|\partial_{t} v_{n}^{\varepsilon}(t)\right\|^{2},
\end{aligned}
$$

where the last inequality comes from the Hölder inequality and (6).

Estimate $v_{n}^{\varepsilon}$ in $L^{\infty}\left(0, T ; H^{1}(\Omega)\right)$ and $\partial_{t} v_{n}^{\varepsilon}$ in $L^{\infty}\left(0, T ; L^{2}(\Omega)\right)$. It follows from (21) that

$$
\begin{aligned}
& \partial_{t}\left(\frac{\left\|\partial_{t} v_{n}^{\varepsilon}(t)\right\|^{2}}{\rho-1}+\rho\left\|v_{n}^{\varepsilon}(t)\right\|^{2}+\left\|\nabla v_{n}^{\varepsilon}(t)\right\|^{2}\right) \\
& \geq 2 C_{1} \log (\gamma) \rho\left(\frac{\left\|\partial_{t} v_{n}^{\varepsilon}(t)\right\|^{2}}{\rho-1}+\rho\left\|v_{n}^{\varepsilon}(t)\right\|^{2}+\left\|\nabla v_{n}^{\varepsilon}(t)\right\|^{2}\right),
\end{aligned}
$$


By Grönwall's inequality, we get

$$
\begin{aligned}
& \frac{\left\|\partial_{t} v_{n}^{\varepsilon}(t)\right\|^{2}}{\rho-1}+\rho\left\|v_{n}^{\varepsilon}(t)\right\|^{2}+\left\|\nabla v_{n}^{\varepsilon}(t)\right\|^{2} \\
& \leq\left(\frac{\left\|\partial_{t} v_{n}^{\varepsilon}(T)\right\|^{2}}{\rho-1}+\rho\left\|v_{n}^{\varepsilon}(T)\right\|^{2}+\left\|\nabla v_{n}^{\varepsilon}(T)\right\|^{2}\right) \gamma^{2 C_{1} \rho(T-t)} .
\end{aligned}
$$

From (20), one gets

$$
\left\{\begin{array}{l}
\partial_{t} v_{n}^{\varepsilon} \text { is uniformly bounded in } L^{\infty}\left(0, T ; L^{2}(\Omega)\right), \\
v_{n}^{\varepsilon} \text { is uniformly bounded in } L^{\infty}\left(0, T ; H^{1}(\Omega)\right) .
\end{array}\right.
$$

It follows from the Banach-Alaoglu theorem, and the argument that a weak limit of derivative is the derivative of the weak limit, that we can extract a subsequence of scaled approximate solutions $v_{n}^{\varepsilon}$, which we still denote by $\left\{v_{n}^{\varepsilon}\right\}_{n \in \mathbb{N}}$, such that for each $\varepsilon>0$

$$
\left\{\begin{array}{l}
\partial_{t} v_{n}^{\varepsilon} \rightarrow \partial_{t} v^{\varepsilon} \text { weakly }-* \text { in } L^{\infty}\left(0, T ; L^{2}(\Omega)\right), \\
v_{n}^{\varepsilon} \rightarrow v^{\varepsilon} \text { weakly }-* \text { in } L^{\infty}\left(0, T ; H^{1}(\Omega)\right) .
\end{array}\right.
$$

Estimate $\partial_{t} v_{n}^{\varepsilon}$ in $L^{2}\left(0, T ; H_{0}^{1}(\Omega)\right)$. Integrating both sides of (21) from 0 to $T$, we get

$$
\begin{aligned}
& (2 \rho-1)\left\|\partial_{t} v_{n}^{\varepsilon}\right\|_{L^{2}\left(0, T ; L^{2}(\Omega)\right)}^{2}+\left\|\nabla v_{n}^{\varepsilon}\right\|_{L^{2}\left(0, T ; L^{2}(\Omega)\right)}^{2} \\
& \leq C_{1} \log (\gamma)\left((\rho-1)\left\|v_{n}^{\varepsilon}\right\|_{L^{2}\left(0, T ; H_{0}^{1}(\Omega)\right.}^{2}+\rho\left\|\partial_{t} v_{n}^{\varepsilon}\right\|_{L^{2}\left(0, T ; L^{2}(\Omega)\right.}^{2}\right) \\
& +\frac{1}{2}\left[\left\|\partial_{t} v_{n}^{\varepsilon}(T)\right\|^{2}+\left(\rho^{2}-\rho\right)\left\|v_{n}^{\varepsilon}(T)\right\|^{2}+(\rho-1)\left\|\nabla v_{n}^{\varepsilon}(T)\right\|^{2}\right] .
\end{aligned}
$$

From (20) and (23), it is straightforward to see that

$$
\left\|\partial_{t} v_{n}^{\varepsilon}\right\|_{L^{2}\left(0, T ; H_{0}^{1}(\Omega)\right)} \leq \bar{C} \quad \text { for all } n \in \mathbb{N} .
$$

for some constant $\bar{C}$.

Estimate $\partial_{t t} v_{n}^{\varepsilon}$ in $L^{2}\left(0, T ; H^{-1}(\Omega)\right)$. Let $\mathbb{S}_{n}$ be a closed subspace of $H_{0}^{1}(\Omega)$ defined by $\mathbb{S}_{n}=\left\{\varphi \in H_{0}^{1}(\Omega): \int_{\Omega} \varphi \varphi_{k} d x=0\right.$ for all $\left.k \leq n\right\}$. Let $\mathbb{S}_{n}^{\perp}$ be a closed subspace of $H_{0}^{1}(\Omega)$ such that $H_{0}^{1}(\Omega)=\mathbb{S}_{n} \oplus \mathbb{S}_{n}^{\perp}$. In other words, for all $\varphi \in H_{0}^{1}(\Omega)$, we can write $\varphi$ of the form $\varphi=\varphi_{n}+\varphi_{n}^{\perp}$ where $\varphi \in \mathbb{S}_{n}$ and 
$\varphi_{n}^{\perp} \in \mathbb{S}_{n}^{\perp}$. Therefore, for a.e. $t \in[0, T]$, from (17), one gets

$$
\begin{aligned}
& \left\langle\partial_{t t} v_{n}^{\varepsilon}(t), \varphi\right\rangle \\
& =(2 \rho-1)\left\langle\partial_{t} v_{n}^{\varepsilon}(t), \varphi_{n}\right\rangle+\left(\rho-\rho^{2}\right)\left\langle v_{n}^{\varepsilon}(t), \varphi_{n}\right\rangle+(1-\rho)\left\langle\nabla v_{n}^{\varepsilon}(t), \nabla \varphi_{n}\right\rangle \\
& +\left\langle\nabla \partial_{t} v_{n}^{\varepsilon}, \nabla \varphi_{n}\right\rangle+(1-\rho)\left\langle\mathbf{P}_{\varepsilon}\left(v_{n}^{\varepsilon}(t)\right), \varphi_{n}\right\rangle+\left\langle\mathbf{P}_{\varepsilon}\left(\partial_{t} v_{n}^{\varepsilon}(t)\right), \varphi_{n}\right\rangle \\
& \leq(2 \rho-1)\left\|\partial_{t} v_{n}^{\varepsilon}(t)\right\|\left\|\varphi_{n}\right\|+\left(\rho^{2}-\rho\right)\left\|v_{n}^{\varepsilon}(t)\right\|\left\|\varphi_{n}\right\| \\
& +(\rho-1)\left\|\nabla v_{n}^{\varepsilon}(t)\right\|\left\|\nabla \varphi_{n}\right\|+\left\|\partial_{t} \nabla v_{n}^{\varepsilon}(t)\right\|\left\|\nabla \varphi_{n}\right\| \\
& +(\rho-1)\left\|\mathbf{P}_{\varepsilon}\left(v_{n}^{\varepsilon}(t)\right)\right\|\left\|\varphi_{n}\right\|+\left\|\mathbf{P}_{\varepsilon}\left(\partial_{t} v_{n}^{\varepsilon}(t)\right)\right\|\left\|\varphi_{n}\right\| .
\end{aligned}
$$

Since $\left\|\varphi_{n}\right\|_{H_{0}^{1}(\Omega)} \leq\left\|\varphi_{n}\right\|_{H_{0}^{1}(\Omega)}+\left\|\varphi_{n}^{\perp}\right\|_{H_{0}^{1}(\Omega)}=\|\varphi\|_{H_{0}^{1}(\Omega)}$ for all $n \in \mathbb{N}$, we get

$$
\begin{aligned}
& \left\|\partial_{t t} v_{n}^{\varepsilon}(t)\right\|_{H^{-1}(\Omega)}=\sup _{\varphi \in H_{0}^{1}(\Omega) \backslash\{0\}} \frac{\left\langle\partial_{t t} v_{n}^{\varepsilon}(t), \varphi\right\rangle}{\|\varphi\|_{H_{0}^{1}(\Omega)}} \\
& \leq(2 \rho-1)\left\|\partial_{t} v_{n}^{\varepsilon}(t)\right\|+\left(\rho^{2}-\rho\right)\left\|v_{n}^{\varepsilon}(t)\right\|+(\rho-1)\left\|\nabla v_{n}^{\varepsilon}(t)\right\| \\
& +\left\|\partial_{t} \nabla v_{n}^{\varepsilon}(t)\right\|+C_{1} \log (\gamma)\left((1-\rho)\left\|v_{n}^{\varepsilon}(t)\right\|_{H_{0}^{1}(\Omega)}+\left\|\partial_{t} v_{n}^{\varepsilon}(t)\right\|_{H_{0}^{1}(\Omega)}\right),
\end{aligned}
$$

where the last term in the right-hand side comes from the properties of $\mathbf{P}_{\varepsilon}^{1}$ and $\mathbf{P}_{\varepsilon}^{2}$. From (23) and (25), there exists a constant $\tilde{C}>0$ such that

$$
\left\|\partial_{t t} v_{n}^{\varepsilon}\right\|_{L^{2}\left(0, T ; H^{-1}(\Omega)\right)} \leq \tilde{C} \quad \text { for all } n \in \mathbb{N} .
$$

Henceforth, from the Banach-Alaoglu theorem, there exists a subsequence of $\left\{v_{n}^{\varepsilon}\right\}$ (still denoted by $\left\{v_{n}^{\varepsilon}\right\}$ ) such that

$$
\partial_{t t} v_{n}^{\varepsilon} \rightarrow \partial_{t t} v^{\varepsilon} \text { weakly in } L^{2}\left(0, T ; H^{-1}(\Omega)\right) .
$$

Combining the above weak-star and weak limits, the function $v^{\varepsilon}$ satisfies

$$
\left\{\begin{array}{l}
v^{\varepsilon} \in L^{\infty}\left(0, T ; H_{0}^{1}(\Omega)\right), \\
\partial_{t} v^{\varepsilon} \in L^{\infty}\left(0, T ; L^{2}(\Omega)\right) \cap L^{2}\left(0, T ; H_{0}^{1}(\Omega)\right), \\
\partial_{t t} v^{\varepsilon} \in L^{2}\left(0, T ; H^{-1}(\Omega)\right) .
\end{array}\right.
$$

Furthermore, since $H_{0}^{1}(\Omega)$ is compactly embedded in $L^{2}(\Omega)$ and $L^{2}(\Omega)$ is continuously embedded in $H^{-1}(\Omega)$ (by Rellich-Kondrachov), from Aubin-Lions lemma, we get

$$
\left\{\begin{array}{l}
v_{n}^{\varepsilon} \rightarrow v^{\varepsilon} \text { strongly in } C\left([0, T] ; H_{0}^{1}(\Omega)\right), \\
\partial_{t} v_{n}^{\varepsilon} \rightarrow \partial_{t} v^{\varepsilon} \text { strongly in } C\left([0, T] ; L^{2}(\Omega)\right) .
\end{array}\right.
$$


Fix an integer $N$ and choose a function $\bar{v} \in C^{1}\left(0, T ; H_{0}^{1}(\Omega)\right)$ having the form

$$
\bar{v}(t)=\sum_{k=1}^{N} d_{k}(t) \phi_{k}
$$

where $d_{1}, \ldots, d_{N}$ are given real valued $C^{1}$ functions defined in $[0, T]$. For all $x \geq N$, multiplying (17), summing for $k=1, \ldots, N$ and integrating over $(0, T)$ lead to

$$
\begin{aligned}
& \int_{\Omega} \partial_{t t} v_{n}^{\varepsilon}(t) \bar{v} d x+(1-2 \rho) \int_{\Omega} \partial_{t} v_{n}^{\varepsilon}(t) \bar{v} d x+\left(\rho^{2}-\rho\right) \int_{\Omega} v_{n}^{\varepsilon}(t) \bar{v} d x \\
& +(\rho-1) \int_{\Omega} \nabla v_{n}^{\varepsilon}(t) \cdot \nabla \bar{v} d x-\int_{\Omega} \nabla \partial_{t} v_{n}^{\varepsilon}(t) \cdot \nabla \bar{v} d x \\
& =(1-\rho) \int_{\Omega} \mathbf{P}_{\varepsilon} v_{n}^{\varepsilon}(t) \bar{v} d x+\int_{\Omega} \mathbf{P}_{\varepsilon} \partial_{t} v_{n}^{\varepsilon}(t) \bar{v} d x .
\end{aligned}
$$

Letting $n \rightarrow \infty$, we obtain from (28) that

$$
\begin{aligned}
& \int_{\Omega} \partial_{t t} v^{\varepsilon}(t) \bar{v} d x+(1-2 \rho) \int_{\Omega} \partial_{t} v^{\varepsilon}(t) \bar{v} d x+\left(\rho^{2}-\rho\right) \int_{\Omega} v^{\varepsilon}(t) \bar{v} d x \\
& +(\rho-1) \int_{\Omega} \nabla v^{\varepsilon}(t) \cdot \nabla \bar{v} d x-\int_{\Omega} \nabla \partial_{t} v^{\varepsilon}(t) \cdot \nabla \bar{v} d x \\
& =(1-\rho) \int_{\Omega} \mathbf{P}_{\varepsilon} v^{\varepsilon}(t) \bar{v} d x+\int_{\Omega} \mathbf{P}_{\varepsilon} \partial_{t} v^{\varepsilon}(t) \bar{v} d x .
\end{aligned}
$$

Since the functions of the form (29) are dense in $L^{2}\left(0, T ; H_{0}^{1}(\Omega)\right)$, the equality (30) holds for all test function $\bar{v} \in L^{2}\left(0, T ; H_{0}^{1}(\Omega)\right)$. We deduce that the function $v^{\varepsilon}$ obtained from approximate solutions $v_{n}^{\varepsilon}$ satisfies the weak formulation in Definition 3.2.

It now remains to verify the initial data for $v^{\varepsilon}$. Take $\kappa \in C^{1}([0, T])$ satisfying $\kappa(T)=1$ and $\kappa(0)=0$. It follows from (24) that

$$
\int_{0}^{T}\left\langle\partial_{t} v_{n}^{\varepsilon}(t), \phi\right\rangle \kappa(t) d t \rightarrow \int_{0}^{T}\left\langle\partial_{t} v^{\varepsilon}(t), \phi\right\rangle \kappa(t) d t \quad \text { for all } \phi \in H_{0}^{1}(\Omega) .
$$

Then by integration by parts, one gets

$$
\begin{aligned}
\int_{0}^{T}\left\langle v_{n}^{\varepsilon}(t), \phi\right\rangle \partial_{t} \kappa(t) d t & -\left\langle v_{n}^{\varepsilon}(T), \phi\right\rangle \kappa(T) \\
& \rightarrow \int_{0}^{T}\left\langle v^{\varepsilon}(t), \phi\right\rangle \partial_{t} \kappa(t) d t-\left\langle v^{\varepsilon}(T), \phi\right\rangle \kappa(T)
\end{aligned}
$$


and thereupon, we get $\left\langle v_{n}^{\varepsilon}(T), \phi\right\rangle \rightarrow\left\langle v^{\varepsilon}(T), \phi\right\rangle$ for all $\phi \in H_{0}^{1}(\Omega)$ by virtue of (24). From Lemma 3.3, we also have that $v_{n}^{\varepsilon}(T) \rightarrow f_{0}^{\varepsilon}$ in $L^{2}(\Omega)$ as $n \rightarrow \infty$. Thus $\left\langle v^{\varepsilon}(T), \phi\right\rangle=\left\langle f_{0}^{\varepsilon}, \phi\right\rangle$ for all $\phi \in H_{0}^{1}(\Omega)$, which implies that $v^{\varepsilon}(T)=f_{0}^{\varepsilon}$ a.e. in $\Omega$. Similarly, it follows from (27) that

$$
\int_{0}^{T}\left\langle\partial_{t t} v_{n}^{\varepsilon}(t), \phi\right\rangle \kappa(t) d t \rightarrow \int_{0}^{T}\left\langle\partial_{t t} v^{\varepsilon}(t), \phi\right\rangle \kappa(t) d t \quad \text { for all } \phi \in H_{0}^{1}(\Omega) .
$$

Then by integration by parts, one gets

$$
\begin{aligned}
& -\int_{0}^{T}\left\langle\partial_{t} v_{n}^{\varepsilon}(t), \phi\right\rangle \partial_{t} \kappa(t) d t+\left\langle\partial_{t} v_{n}^{\varepsilon}(T), \phi\right\rangle \kappa(T) \\
& \rightarrow-\int_{0}^{T}\left\langle\partial_{t} v^{\varepsilon}(t), \phi\right\rangle \partial_{t} \kappa(t) d t+\left\langle\partial_{t} v^{\varepsilon}(T), \phi\right\rangle \kappa(T) \quad \text { as } n \rightarrow \infty .
\end{aligned}
$$

Using the similar arguments as in the proof for $v^{\varepsilon}(T)$, we obtain that $\partial_{t} v^{\varepsilon}(T)=$ $\rho f_{0}^{\varepsilon}+f_{1}^{\varepsilon}$ a.e. in $\Omega$. Hence, we complete the proof of the existence.

Finally, we are going to prove the uniqueness of (12)-(13). We sketch out some important steps because this proof is standard. Indeed, let $v^{\varepsilon}$ and $\bar{v}^{\varepsilon}$ be two weak solutions of the system (12)-(13). Since the system is linear, it is straightforward to see that the function $k^{\varepsilon}=v^{\varepsilon}-\bar{v}^{\varepsilon}$ satisfies (12) with zero terminal conditions $k^{\varepsilon}(T)=\partial_{t} k^{\varepsilon}(T)=0$. Taking $\varphi=\partial_{t} k^{\varepsilon}$ as a test function, we proceed as in the way to get the estimate (22). Hence, $k^{\varepsilon}(t)=0$ a.e. in $(0, T)$ because of the fact that

$$
\frac{\left\|\partial_{t} k^{\varepsilon}(t)\right\|^{2}}{\rho-1}+\rho\left\|k^{\varepsilon}(t)\right\|^{2}+\left\|\nabla k^{\varepsilon}(t)\right\|^{2} \leq 0 \quad \text { a.e. in }(0, T) .
$$

This completes the proof of the theorem.

\section{Convergence analysis}

In this part, our focus is on the convergence analysis of the variational QR framework adapted to solve the time-reversed hyperbolic heat conduction problem. The error estimate obtained below can be viewed as a "worst-case" scenario of convergence of this QR scheme in case the stabilized operator $\mathbf{P}_{\varepsilon}$ is bounded logarithmically.

It is worth noting that our analysis in section 3 does not care about the dependence of $C$ (and any type of constants in there) on the noise level $\varepsilon$, since basically we fix $\varepsilon$. However, to this end any constant $C>0$ used below should be $\varepsilon$ independent because we are going to show the error estimates with respect to only $\varepsilon$. 
Theorem 4.1. Assume (9) holds. Let $\varepsilon \in(0,1)$ be a sufficiently small number such that $\gamma:=\gamma(\varepsilon) \geq e^{2 / C_{1}}$. Suppose the following conditions hold

$$
\left\{\begin{array}{l}
3 C_{1} T<2, \\
\lim _{\varepsilon \rightarrow 0} \gamma^{2}(\varepsilon) \varepsilon \leq K .
\end{array}\right.
$$

Next, assume the original system (2)-(4) admits a unique solution $u$ such that $u \in C([0, T] ; \mathbb{W})$ and $u_{t} \in L^{2}(0, T ; \mathbb{W})$, where $\mathbb{W}$ is obtained in Definition 2.1. Let $M>0$ be such that

$$
\|u\|_{C([0, T] ; \mathbb{W})}^{2}+\left\|u_{t}\right\|_{L^{2}(0, T ; \mathbb{W})}^{2} \leq M .
$$

Let $u^{\varepsilon}$ be a unique weak solution of the regularized system (7)-(8) analyzed in Theorem 3.5. Then for $0 \leq t \leq T$ the following error estimates hold:

$$
\begin{aligned}
& \left\|u^{\varepsilon}(t)-u(t)\right\|^{2} \leq C\left(\varepsilon+(\log (\gamma))^{-1} \gamma^{3 C_{1}(T-t)-2}\right), \\
& \left\|\nabla u^{\varepsilon}(t)-\nabla u(t)\right\|^{2} \leq C\left(\log (\gamma) \varepsilon+\gamma^{3 C_{1}(T-t)-2}\right) \\
& \left\|u_{t}^{\varepsilon}(t)-u_{t}(t)\right\|^{2}+\int_{t}^{T}\left\|\nabla u_{t}^{\varepsilon}(s)-\nabla u_{t}(s)\right\|^{2} d s \\
& \leq C\left((\log (\gamma))^{2} \varepsilon+\log (\gamma) \gamma^{3 C_{1}(T-t)-2}\right) .
\end{aligned}
$$

where $C=C\left(K, M, C_{0}, C_{1}\right)>0$ is independent of $\varepsilon$.

Proof. Let $w^{\varepsilon}(x, t)=\left[u^{\varepsilon}(x, t)-u(x, t)\right] e^{\rho_{\varepsilon}(t-T)}$ for some $\rho_{\varepsilon}>0$, viewing as a weighted difference function in our proof of convergence. The notion behind this use of the Carleman weight function is to "maximize" the measured terminal data that we are having and thus, we can take full advantage of the noise level $\varepsilon$. The weight function here is classical in the framework of parabolic equations backward in time; cf. e.g. [28, Section 9]. In principle, the downscaling (with respect to the noise level) used here is helpful in getting rid of the large stability magnitude by a suitable choice of the auxiliary parameter $\rho_{\varepsilon}$, which is also relatively large. Now, we compute the equation for $w^{\varepsilon}$, calling as the difference equation between the regularized problem (7)-(8) and the original system (2)-(4). In fact, we have

$$
\begin{aligned}
w_{t}^{\varepsilon} & =\left[u_{t}^{\varepsilon}-u_{t}\right] e^{\rho_{\varepsilon}(t-T)}+\rho_{\varepsilon}\left[u^{\varepsilon}-u\right] e^{\rho_{\varepsilon}(t-T)} \\
& =\left[u_{t}^{\varepsilon}-u_{t}\right] e^{\rho_{\varepsilon}(t-T)}+\rho_{\varepsilon} w^{\varepsilon}, \\
\Delta w^{\varepsilon} & =\left[\Delta u^{\varepsilon}-\Delta u\right] e^{\rho_{\varepsilon}(t-T)},
\end{aligned}
$$


which lead to

$$
\begin{aligned}
w_{t t}^{\varepsilon}-\rho_{\varepsilon} w_{t}^{\varepsilon} & =\left[u_{t t}^{\varepsilon}-u_{t t}\right] e^{\rho_{\varepsilon}(t-T)}+\rho_{\varepsilon}\left[u_{t}^{\varepsilon}-u_{t}\right] e^{\rho_{\varepsilon}(t-T)} \\
& =\left[u_{t t}^{\varepsilon}-u_{t t}\right] e^{\rho_{\varepsilon}(t-T)}+\rho_{\varepsilon}\left(w_{t}^{\varepsilon}-\rho_{\varepsilon} w^{\varepsilon}\right), \\
\Delta w_{t}^{\varepsilon}-\rho_{\varepsilon} \Delta w^{\varepsilon} & =\left[\Delta u_{t}^{\varepsilon}-\Delta u_{t}\right] e^{\rho_{\varepsilon}(t-T)} .
\end{aligned}
$$

Hereby, we notice that when multiplying both sides of the systems (7)-(8) and (2)-(4) by the weight $e^{\rho_{\varepsilon}(t-T)}$, it yields

$$
\begin{aligned}
& {\left[u_{t t}^{\varepsilon}-u_{t t}\right] e^{\rho_{\varepsilon}(t-T)}+\left[u_{t}^{\varepsilon}-u_{t}\right] e^{\rho_{\varepsilon}(t-T)}+\Delta\left(u^{\varepsilon}-u\right) e^{\rho_{\varepsilon}(t-T)}} \\
& +\Delta\left(u_{t}^{\varepsilon}-u_{t}\right) e^{\rho_{\varepsilon}(t-T)}=\mathbf{P}_{\varepsilon}\left(u^{\varepsilon}-u\right) e^{\rho_{\varepsilon}(t-T)}+\mathbf{Q}_{\varepsilon} u e^{\rho_{\varepsilon}(t-T)} \\
& +\mathbf{P}_{\varepsilon}\left(u_{t}^{\varepsilon}-u_{t}\right) e^{\rho_{\varepsilon}(t-T)}+\mathbf{Q}_{\varepsilon} u_{t} e^{\rho_{\varepsilon}(t-T)} .
\end{aligned}
$$

Henceforth, we plug the identities (32)-(35) into the equation (36) to get

$$
\begin{aligned}
& w_{t t}^{\varepsilon}+\left(\rho_{\varepsilon}^{2}-\rho_{\varepsilon}\right) w^{\varepsilon}-\left(\rho_{\varepsilon}-1\right) \Delta w^{\varepsilon}+\Delta w_{t}^{\varepsilon} \\
& =\mathbf{P}_{\varepsilon} w^{\varepsilon}+\mathbf{Q}_{\varepsilon} u e^{\rho_{\varepsilon}(t-T)}+\left(2 \rho_{\varepsilon}-1\right) w_{t}^{\varepsilon}+\mathbf{P}_{\varepsilon}\left(w_{t}^{\varepsilon}-\rho_{\varepsilon} w^{\varepsilon}\right)+\mathbf{Q}_{\varepsilon} u_{t} e^{\rho_{\varepsilon}(t-T)} .
\end{aligned}
$$

which is the PDE for the difference function $w^{\varepsilon}$.

Now, we multiply both sides of (37) by $w_{t}^{\varepsilon}$ and integrate the resulting equation over $\Omega$. After some manipulations, we arrive at

$$
\begin{aligned}
& \frac{1}{2} \frac{d}{d t}\left\|w_{t}^{\varepsilon}\right\|^{2}+\frac{1}{2}\left(\rho_{\varepsilon}^{2}-\rho_{\varepsilon}\right) \frac{d}{d t}\left\|w^{\varepsilon}\right\|^{2}+\frac{1}{2}\left(\rho_{\varepsilon}-1\right) \frac{d}{d t}\left\|\nabla w^{\varepsilon}\right\|^{2}-\left\|\nabla w_{t}^{\varepsilon}\right\|^{2} \\
& =\left(2 \rho_{\varepsilon}-1\right)\left\|w_{t}^{\varepsilon}\right\|^{2}+\left\langle\left(\mathbf{P}_{\varepsilon}-\rho_{\varepsilon} \mathbf{P}_{\varepsilon}\right) w^{\varepsilon}, w_{t}^{\varepsilon}\right\rangle+\left\langle\mathbf{P}_{\varepsilon} w_{t}^{\varepsilon}, w_{t}^{\varepsilon}\right\rangle \\
& +e^{\rho_{\varepsilon}(t-T)}\left\langle\mathbf{Q}_{\varepsilon} u, w_{t}^{\varepsilon}\right\rangle+e^{\rho_{\varepsilon}(t-T)}\left\langle\mathbf{Q}_{\varepsilon} u_{t}, w_{t}^{\varepsilon}\right\rangle .
\end{aligned}
$$

Based upon the conditional estimates (5)-(6) we estimate the right-hand side of (38) as follows:

$$
\begin{aligned}
& \left\langle\left(\mathbf{P}_{\varepsilon}-\rho_{\varepsilon} \mathbf{P}_{\varepsilon}\right) w^{\varepsilon}, w_{t}^{\varepsilon}\right\rangle \geq-\frac{1}{2}\left(\rho_{\varepsilon}-1\right) C_{1}^{2}(\log (\gamma))^{2}\left\|w^{\varepsilon}\right\|^{2}-\frac{1}{2}\left(\rho_{\varepsilon}-1\right)\left\|w_{t}^{\varepsilon}\right\|^{2}, \\
& \left\langle\mathbf{P}_{\varepsilon} w_{t}^{\varepsilon}, w_{t}^{\varepsilon}\right\rangle \geq-C_{1} \log (\gamma)\left\|w_{t}^{\varepsilon}\right\|^{2} \\
& e^{\rho_{\varepsilon}(t-T)}\left\langle\mathbf{Q}_{\varepsilon} u, w_{t}^{\varepsilon}\right\rangle \geq-\frac{1}{2}\left(\frac{1}{4}\left\|w_{t}^{\varepsilon}\right\|^{2}+4 e^{2 \rho_{\varepsilon}(t-T)} C_{0}^{2} \gamma^{-2}\|u\|_{\mathbb{W}_{1}}^{2}\right) \\
& e^{\rho_{\varepsilon}(t-T)}\left\langle\mathbf{Q}_{\varepsilon} u_{t}, w_{t}^{\varepsilon}\right\rangle \geq-\frac{1}{2}\left(\frac{1}{4}\left\|w_{t}^{\varepsilon}\right\|^{2}+4 e^{2 \rho_{\varepsilon}(t-T)} C_{0}^{2} \gamma^{-2}\left\|u_{t}\right\|_{\mathbb{W}_{2}}^{2}\right)
\end{aligned}
$$


Therefore, by integrating (38) from $t$ to $T$ we estimate that

$$
\begin{aligned}
& \left\|w_{t}^{\varepsilon}(t)\right\|^{2}+\left(\rho_{\varepsilon}^{2}-\rho_{\varepsilon}\right)\left\|w^{\varepsilon}(t)\right\|^{2}+\left(\rho_{\varepsilon}-1\right)\left\|\nabla w^{\varepsilon}(t)\right\|^{2}+2 \int_{t}^{T}\left\|\nabla w_{t}^{\varepsilon}(s)\right\|^{2} d s \\
& \leq\left\|w_{t}^{\varepsilon}(T)\right\|^{2}+\left(\rho_{\varepsilon}^{2}-\rho_{\varepsilon}\right)\left\|w^{\varepsilon}(T)\right\|^{2}+\left(\rho_{\varepsilon}-1\right)\left\|\nabla w^{\varepsilon}(T)\right\|^{2} \\
& +4 C_{0}^{2} \gamma^{-2} \rho_{\varepsilon}^{-1}\left(1-e^{2 \rho_{\varepsilon}(t-T)}\right)\|u\|_{C\left([0, T] ; \mathbb{W}_{1}\right)}^{2}+4 C_{0}^{2} \gamma^{-2}\left\|u_{t}\right\|_{L^{2}\left(0, T ; \mathbb{W}_{2}\right)}^{2} \\
& +C_{1}^{2} \rho_{\varepsilon}^{-1}(\log (\gamma))^{2} \int_{t}^{T} \rho_{\varepsilon}\left(\rho_{\varepsilon}-1\right)\left\|w^{\varepsilon}(s)\right\|^{2} d s \\
& +2\left[\frac{1}{2}\left(\rho_{\varepsilon}-1\right)+C_{1} \log (\gamma)+\frac{1}{2}-2 \rho_{\varepsilon}+1\right] \int_{t}^{T}\left\|w_{t}^{\varepsilon}(s)\right\|^{2} d s .
\end{aligned}
$$

By choosing $\rho_{\varepsilon}=C_{1} \log (\gamma) \geq 2$ (since $\gamma \geq e^{2 / C_{1}}$ ), the last term in the right-hand side becomes $\left(2-\rho_{\varepsilon}\right) \int_{t}^{T}\left\|w_{t}^{\varepsilon}(s)\right\|^{2} d s \leq 0$, we apply the Grönwall inequality to obtain

$$
\begin{aligned}
& \left\|w_{t}^{\varepsilon}(t)\right\|^{2}+\left(\rho_{\varepsilon}^{2}-\rho_{\varepsilon}\right)\left\|w^{\varepsilon}(t)\right\|^{2}+\left(\rho_{\varepsilon}-1\right)\left\|\nabla w^{\varepsilon}(t)\right\|^{2}+2 \int_{t}^{T}\left\|\nabla w_{t}^{\varepsilon}(s)\right\|^{2} d s \\
& \leq\left[2\left(\rho_{\varepsilon}^{2}+1\right) \varepsilon^{2}+\varepsilon^{2}\left(\rho_{\varepsilon}^{2}-1\right)+4 C_{0}^{2} \gamma^{-2} M\right] \gamma^{C_{1}(T-t)},
\end{aligned}
$$

where we have used the measurement assumption (9) and the fact that

$$
\left\|w_{t}^{\varepsilon}(T)\right\|^{2}=\left\|\left[u_{t}^{\varepsilon}(T)-u_{t}(T)\right]+\rho_{\varepsilon}\left[u^{\varepsilon}(T)-u(T)\right]\right\|^{2} \leq 2\left(\rho_{\varepsilon}^{2}+1\right) \varepsilon^{2} .
$$

Thus, using the back-substitution

$$
w^{\varepsilon}(x, t)=\left[u^{\varepsilon}(x, t)-u(x, t)\right] e^{\rho_{\varepsilon}(t-T)}=\left[u^{\varepsilon}(x, t)-u(x, t)\right] \gamma^{C_{1}(t-T)},
$$

we conclude the convergence in $L^{2}(\Omega)$ type as follows:

$$
\begin{aligned}
& \left\|u^{\varepsilon}(t)-u(t)\right\|^{2} \\
& \leq\left(\frac{2\left(\rho_{\varepsilon}^{2}+1\right)}{\rho_{\varepsilon}^{2}-\rho_{\varepsilon}}+\frac{\rho_{\varepsilon}^{2}-1}{\rho_{\varepsilon}^{2}-\rho_{\varepsilon}}\right) \varepsilon^{2} \gamma^{3 C_{1}(T-t)}+\frac{4}{\rho_{\varepsilon}^{2}-\rho_{\varepsilon}} C_{0}^{2} M \gamma^{-2} \gamma^{3 C_{1}(T-t)} \\
& \leq \frac{\rho_{\varepsilon}^{2}-1}{\rho_{\varepsilon}^{2}-\rho_{\varepsilon}}\left(3 \gamma^{3 C_{1}(T-t)}+\gamma^{3 C_{1}(T-t)}\right) \varepsilon^{2}+4 C_{0}^{2} M \rho_{\varepsilon}^{-1} \gamma^{3 C_{1}(T-t)-2} \\
& \leq 2\left(4 \gamma^{3 C_{1}(T-t)} \varepsilon^{2}+C_{1}^{-1} 2 C_{0}^{2} M(\log (\gamma))^{-1} \gamma^{3 C_{1}(T-t)-2}\right) .
\end{aligned}
$$

From (31), we get $\gamma^{3 C_{1}(T-t)} \varepsilon^{2} \leq K^{\frac{3 C_{1} T}{2}} \varepsilon$ and it follows from the previous inequality that

$$
\left\|u^{\varepsilon}(t)-u(t)\right\|^{2} \leq C\left(\varepsilon+(\log (\gamma))^{-1} \gamma^{3 C_{1}(T-t)-2}\right),
$$


for some constant $C>0$. In the same manner, we derive from (42) the convergence for the gradient terms:

$$
\begin{aligned}
\left\|\nabla u^{\varepsilon}(t)-\nabla u(t)\right\|^{2} & \leq 2\left(4 C_{1} \log (\gamma) \gamma^{3 C_{1}(T-t)} \varepsilon^{2}+2 C_{0} M \gamma^{3 C_{1}(T-t)-2}\right) \\
& \leq C\left(\log (\gamma) \varepsilon+\gamma^{3 C_{1}(T-t)-2}\right) .
\end{aligned}
$$

Now using the back-substitution (44), we get

$$
\nabla w_{t}^{\varepsilon}(t)=\left[\nabla u_{t}^{\varepsilon}(t)-\nabla u_{t}(t)\right] \gamma^{C_{1}(t-T)}+\rho_{\varepsilon}\left[\nabla u^{\varepsilon}(t)-\nabla u(t)\right] \gamma^{C_{1}(t-T)} .
$$

It yields

$$
\begin{aligned}
& 2 \int_{t}^{T}\left\|\nabla w_{t}^{\varepsilon}(s)\right\|^{2} d s+2 \int_{t}^{T}\left\|\nabla u^{\varepsilon}(s)-\nabla u(s)\right\|^{2} \rho_{\varepsilon}^{2} \gamma^{2 C_{1}(s-T)} d s \\
& \geq \int_{t}^{T}\left\|\nabla u_{t}^{\varepsilon}(s)-\nabla u_{t}(s)\right\|^{2} \gamma^{2 C_{1}(s-T)} d s \\
& \geq \gamma^{2 C_{1}(t-T)} \int_{t}^{T}\left\|\nabla u_{t}^{\varepsilon}(s)-\nabla u_{t}(s)\right\|^{2} d s .
\end{aligned}
$$

Thus it follows from (42) that

$$
\begin{aligned}
& \int_{t}^{T}\left\|\nabla u_{t}^{\varepsilon}(s)-\nabla u_{t}(s)\right\|^{2} d s \\
\leq & \left(4 \rho_{\varepsilon}^{2} \varepsilon^{2}+4 C_{0}^{2} \gamma^{-2} M\right) \gamma^{3 C_{1}(T-t)}+2 \rho_{\varepsilon}^{2} \gamma^{2 C_{1}(t-T)} \int_{t}^{T}\left\|\nabla u^{\varepsilon}(s)-\nabla u(s)\right\|^{2} d s \\
\leq & {\left[4 C_{1}^{2}(\log (\gamma))^{2} \varepsilon^{2} \gamma^{3 C_{1}(T-t)}+4 C_{0}^{2} M \gamma^{3 C_{1}(T-t)-2}\right] } \\
& +C T \rho_{\varepsilon}^{2} \gamma^{2 C_{1}(t-T)}\left(\varepsilon+(\log (\gamma))^{-1} \gamma^{3 C_{1} T-2}\right),
\end{aligned}
$$

where we have used the estimate (45) for the last inequality. This implies

$$
\int_{t}^{T}\left\|\nabla u_{t}^{\varepsilon}(s)-\nabla u_{t}(s)\right\|^{2} d s \leq C\left((\log (\gamma))^{2} \varepsilon+\log (\gamma) \gamma^{3 C_{1}(T-t)-2}\right) .
$$

Finally, using the back-substitution (44), one has

$$
w_{t}^{\varepsilon}(t)=\left[u_{t}^{\varepsilon}(t)-u_{t}(t)\right] \gamma^{C_{1}(t-T)}+\rho_{\varepsilon}\left[u^{\varepsilon}(t)-u(t)\right] \gamma^{C_{1}(t-T)} .
$$

This implies

$$
\left\|u_{t}^{\varepsilon}(t)-u_{t}(t)\right\|^{2} \gamma^{2 C_{1}(t-T)} \leq 2\left\|w_{t}^{\varepsilon}(s)\right\|^{2}+2 \rho_{\varepsilon}^{2}\left\|u^{\varepsilon}(t)-u(t)\right\|^{2} .
$$


Applying the estimate of $\left\|w_{t}^{\varepsilon}\right\|^{2}$ in (42) and $\left\|u^{\varepsilon}(t)-u(t)\right\|^{2}$ in (45), we obtain

$$
\left\|u_{t}^{\varepsilon}(t)-u_{t}(t)\right\|^{2} \leq C\left((\log (\gamma))^{2} \varepsilon+\log (\gamma) \gamma^{3 C_{1}(T-t)-2}\right) .
$$

Hence, we complete the proof of the theorem.

As a by-product of Theorem 4.1, an appropriate choice of $\gamma$ is taken to state the following convergence result with the Hölder rates. It is then noticeable that our error estimates in Theorem 4.1 and in Corollary 4.2 below are uniform in time. In this regard, they are still true for $t=0$ under the restriction (31). This Hölder convergence result is quite different from that of the backward heat equations, which is usually logarithmic at $t=0$ and is of Hölder type at $t>0$; cf. e.g. $[8,9,17]$. In the framework of the backward hyperbolic problems, our convergence result can also be compared with those obtained in [26].

Corollary 4.2. Under the assumptions of Theorem 4.1, if we choose $\gamma(\varepsilon)=\varepsilon^{-1 / 2}$, then for any $\varepsilon \leq e^{-4 / C_{1}}$ the following error estimates hold:

$$
\begin{aligned}
& \left\|u^{\varepsilon}(t)-u(t)\right\|^{2} \leq C\left(\varepsilon+\left(\log \left(\varepsilon^{-1 / 2}\right)\right)^{-1} \varepsilon^{1-3 C_{1}(T-t) / 2}\right), \\
& \left\|\nabla u^{\varepsilon}(t)-\nabla u(t)\right\|^{2} \leq C\left(\log \left(\varepsilon^{-1 / 2}\right) \varepsilon+\varepsilon^{1-3 C_{1}(T-t) / 2}\right), \\
& \left\|u_{t}^{\varepsilon}(t)-u_{t}(t)\right\|^{2}+\int_{t}^{T}\left\|\nabla u_{t}^{\varepsilon}(s)-\nabla u_{t}(s)\right\|^{2} d s \\
& \leq C\left(\left(\log \left(\varepsilon^{-1 / 2}\right)\right)^{2} \varepsilon+\log \left(\varepsilon^{-1 / 2}\right) \varepsilon^{1-3 C_{1}(T-t) / 2}\right) .
\end{aligned}
$$

where $C=C\left(M, C_{0}, C_{1}\right)>0$ is independent of $\varepsilon$.

Remark 4.3. - If we apply the perturbing and stabilized operators (10) and (11) in Remark 2.4 to Theorem 4.1, regularity of the true solution of the original system (2)-(4) is restricted in the Gevrey space. More precisely, one has $u \in C\left([0, T] ; \mathbb{W}_{1,1}\right)$ and $u_{t} \in C\left([0, T] ; \mathbb{W}_{1,1}\right)$ in Theorem 4.1. The same result is applied for Corollary 4.2.

- Obviously, the perturbation for $-\Delta u$ and $-\Delta u_{t}$ of (2) can be different from each other. This will also lead to different regularity assumptions on the exact solution that we have assumed in Theorem 4.1.

- We remark that if the measurement assumption (9) is only given by

$$
\left\|u^{\varepsilon}(\cdot, T)-u(\cdot, T)\right\| \leq \varepsilon,
$$


we obtain the logarithmic rate of convergence in the following sense:

$$
\left\|u^{\varepsilon}(t)-u(t)\right\|^{2} \leq C /(\log (\gamma))^{2} .
$$

Acknowledgments. V.A.K would like to thank Prof. Michael Victor Klibanov (Charlotte, USA) for his wholehearted guidance during the fellowship at UNCC and for giving a chance to delve into the Carleman estimates and convexification.

\section{Bibliography}

[1] M. Asch, M. Bocquet and M. Nodet, Data Assimilation: Methods, Algorithms, and Applications, Society for Industrial and Applied Mathematics, 2016.

[2] S. A. Avdonin, S. A. Ivanov and J. M. Wang, Inverse problems for the heat equation with memory, Inverse Problems \& Imaging 13 (2019), 31-38.

[3] E. Bonetti, E. Rocca, R. Scala and G. Schimperna, On the strongly damped wave equation with constraint, Communications in Partial Differential Equations 42 (2017), 1042-1064.

[4] A. S. Carasso, J. G. Sanderson and J. M. Hyman, Digital removal of random media image degradations by solving the diffusion equation backwards in time, SIAM Journal on Numerical Analysis 15 (1978), 344-367.

[5] C. I. Christov and P. M. Jordan, Heat conduction paradox involving second-sound propagation in moving media, Physical Review Letters 94 (2005).

[6] V. N. Doan, H. T. Nguyen, V. A. Khoa and V. A. Vo, A note on the derivation of filter regularization operators for nonlinear evolution equations, Applicable Analysis 97 (2017), 3-12.

[7] R. E. Ewing, The approximation of certain parabolic equations backward in time by Sobolev equations, SIAM Journal on Mathematical Analysis 6 (1975), 283-294.

[8] Dinh Nho Hào and Nguyen Van Duc, Stability results for the heat equation backward in time, Journal of Mathematical Analysis and Applications 353 (2009), 627-641.

[9] Dinh Nho Hào, Nguyen Van Duc and Nguyen Van Thang, Backward semi-linear parabolic equations with time-dependent coefficients and local Lipschitz source, Inverse Problems 34 (2018), 055010.

[10] R. Jaroudi, G. Baravdish, F. Åström and B. T. Johansson, Source Localization of Reaction-Diffusion Models for Brain Tumors, Lecture Notes in Computer Science, Springer International Publishing, 2016, pp. 414-425.

[11] D. Jou, J. Casas-Vázquez and G. Lebon, Extended Irreversible Thermodynamics, Springer Berlin Heidelberg, 2001. 
[12] S. I. Kabanikhin, Definitions and examples of inverse and ill-posed problems, Journal of Inverse and Ill-posed Problems 16 (2008), 317-357.

[13] B. Kaltenbacher and W. Rundell, Regularization of a backwards parabolic equation by fractional operators, Inverse Problems \& Imaging 13 (2019), 401-430.

[14] M. V. Klibanov, Carleman estimates for the regularization of ill-posed Cauchy problems, Applied Numerical Mathematics 94 (2015), 46-74.

[15] M. V. Klibanov and A. G. Yagola, Convergent numerical methods for parabolic equations with reversed time via a new Carleman estimate, Inverse Problems 35 (2019), 115012.

[16] R. Lattès and J. L. Lions, Méthode de Quasi-réversibilité et Applications, Paris, Dunod, 1967.

[17] N. T. Long and A. P. N. Dinh, Approximation of a parabolic non-linear evolution equation backwards in time, Inverse Problems 10 (1994), 905-914.

[18] N. T. Long and A. P. N. Dinh, Note on a regularization of a parabolic nonlinear evolution equation backwards in time, Inverse Problems 12 (1996), 455-462.

[19] T. N. Luan and T. Q. Khanh, On the backward problem for parabolic equations with memory, Applicable Analysis (2019), 1-18.

[20] V. Méndez and J. Camacho, Dynamics and thermodynamics of delayed population growth, Physical Review E 55 (1997), 6476-6482.

[21] H. T. Nguyen, V. A. Khoa and V. A. Vo, Analysis of a quasi-reversibility method for a terminal value quasi-linear parabolic problem with measurements, SIAM Journal on Mathematical Analysis 51 (2019), 60-85.

[22] S. B. M. Sambatti, H. F. de Campos Velho and L. D. Chiwiacowsky, Epidemic Genetic Algorithm for Solving Inverse Problems: Parallel Algorithms, Integral Methods in Science and Engineering, Springer International Publishing, 2019, pp. 381-394.

[23] R. E. Showalter and T. W. Ting, Pseudoparabolic partial differential equations, SIAM Journal on Mathematical Analysis 1 (1970), 1-26.

[24] N. H. Tuan, V. V. Au, V. A. Khoa and D. Lesnic, Identification of the population density of a species model with nonlocal diffusion and nonlinear reaction, Inverse Problems 33 (2017), 055019.

[25] N. H. Tuan, V. A. Khoa, M. T. N. Truong, T. T. Hung and M. N. Minh, Application of the cut-off projection to solve a backward heat conduction problem in a two-slab composite system, Inverse Problems in Science and Engineering 27 (2018), 460483.

[26] N. H. Tuan, D. V. Nguyen, V. V. Au and D. Lesnic, Recovering the initial distribution for strongly damped wave equation, Applied Mathematics Letters 73 (2017), 69-77. 
[27] J. J. Vadasz, S. Govender and P. Vadasz, Heat transfer enhancement in nano-fluids suspensions: Possible mechanisms and explanations, International Journal of Heat and Mass Transfer 48 (2005), 2673-2683.

[28] Masahiro Yamamoto, Carleman estimates for parabolic equations and applications, Inverse Problems 25 (2009), 123013.

\section{Author information}

Vo Anh Khoa, Department of Mathematics and Statistics, University of North Carolina at Charlotte, Charlotte, North Carolina 28223, USA.

E-mail: vakhoa.hcmus@gmail.com, avo5@uncc.edu

Manh-Khang Dao, Department of Mathematics, KTH Royal Institute of Technology, 100 44 Stockholm, Sweden.

E-mail: mkdao@kth.se 